\title{
Assessment of HICO data for Coastal Studies
}

\author{
Rimjhim Bhatnagar Singh* and Prakash Chauhan \\ Biological and Planetary Sciences and Applications Group, EPSA \\ Space Applications Centre, ISRO, Ahmedabad \\ Corresponding Author, Email: rimjhim@sac.isro.gov.in
}

\begin{abstract}
Coastal waters, in particular, are the regions of high productivity and biodiversity. Detailed investigations of the variability within them can aid in understanding many biogeochemical processes. With the advent of hyperspectral remote sensing having large number of closely spaced channels and highly improved signal-to-noise ratio (SNR), the coastal applications are expected to increase and improve. In India, very less work is done in the field of coastal studies, let alone using hyperspectral remote sensing. HICO, onboard ISS, is the most recent addition to this family of instruments. So, a pilot study was conducted to assess HICO data for coastal studies especially in deriving the shallow water bathymetry estimates. The methodology for deriving bathymetry estimates is based on the different responses of shallow-water reflectance on depth and substrate type because with decreasing water depth in case 2 waters, the spectral contributions arriving from pure water reduce while from other OCAs increase. This variability is typically higher in the wavelength range 480 to $610 \mathrm{~nm}$. Using this wavelength range, bathymetric estimates were made at pixel level. Bathymetry estimates were found to vary from $1 \mathrm{~m}$ to $>12 \mathrm{~m}$. Spectral variability is clearly observed in the continuum removed spectral plots from waters of different depths and is reported in this paper.
\end{abstract}

\section{KEYWORDS}

Hyperspectral, HICO, Bathymetry, Spectra, Optically Complex Water,

\section{INTRODUCTION}

Coastal waters, in particular, are the regions of high productivity and biodiversity. Detailed investigations of the variability within them can aid in understanding many biogeochemical processes such as primary productivity (Cullen et al., 1997), carbon cycle etc and thus in coastal zone management (Nayak et al, 1996). They carry various optically active materials like phytoplankton, NAP, and CDOM. The incident light over water is absorbed and scattered by these materials. Hence, their varied concentration leads to the variability of natural as well as coastal waters and their apparent optical properties (AOPs).

In Case I waters, the optical properties are controlled by phytoplanktons and their associated degradations while in Case II waters which includes inland and coastal waters, their spectral characteristics are influenced by phytoplankton and other OCAs (Morel and Loisel, 1998) including varying proportions of sediments. With decreasing water depth, the spectral contributions arriving from pure water reduce while from other OCAs increase. Using this spectral information, gradients relating to biogeochemical concentrations of various types within water spread may be derived. In the past, many studies related to coastal applications have been done using broad band data. But, the hyperspectral data use for coastal studies is still in the nascent stage in India. With this purview in mind, space-borne hyperspectral data, HICO was investigated for coastal applications.

One of the primary requirements in the field of coastal studies is the bathymetry. Bathymetric or hydrographic charts usually prepared for case 1 waters are suitable for navigation purpose and for oceanic modelling. Presently, deep water bathymetry has a large amount of datasets available with varying coverage, resolutions, and accuracies like ETOPO5 (National Geophysical Data Centre (NGDC) 1988) and the ETOPO2v2 (NGDC 2006) bathymetric grids, both of which are now in wide use. ETOPO2 is based on satellite altimetry and works best in deep-water regions. In shallow waters ETOPO2 is not always reliable (http://www.ngdc.noaa.gov/mgg/global/ relief/ETOPO2/ETOPO2-2001). Hence, it is important to have shallow water bathymetry estimates not only for deriving the spectral characteristics of optically complex waters having variable benthic environment but also for other oceanic applications like studies related to tidal circulation and tsunami propagation. However, shallow water bathymetry is still not as coordinated and standardized as is deep water bathymetry in India as well as internationally. Satellite data based models for shallow water bathymetry mapping have their unique advantages of repetitive and quick quantitative estimation with fewer efforts over large spatial scales (Conger et al, 2006). With the advent of hyperspectral remote sensing having large number of closely spaced channels and highly improved signalto-noise ratio (SNR), coastal studies are going to be more informative. Shallow water bathymetric estimates are expected to improve. Ma et al (2014) have developed a method that can estimate clear shallow-water depth $\left(\begin{array}{lll}0-30 & \mathrm{~m}\end{array}\right)$ from hyperspectral data. In this paper, this method is investigated for deriving coastal bathymetric estimates and thereupon spectral characteristics of waters of varying depths. 


\section{STUDY AREA}

The study was conducted over a coastal part of the Arabian Sea region. The data analyzed was of $16^{\text {th }}$ December 2013. Cloud patches were observed at many places within the scene, however, coastal regions, typically where this study was conducted, were largely cloud free. Figure 1 shows the FCC of the study area.

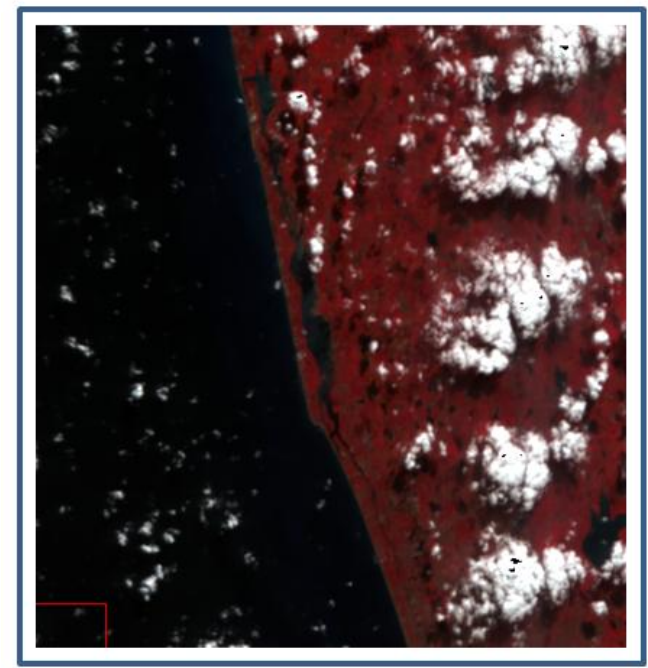

Figure 1: FCC of the study area (Bands selected are band 97, 60 and 42 corresponding to central wavelength $547.28 \mathrm{~nm}, 650.38 \mathrm{~nm}$ and $862.32 \mathrm{~nm}$ respectively)

\section{MATERIALS AND METHODS}

In order to carry out this study, hyperspectral data was obtained from Hyperspectral Imager for Coastal Ocean (HICO). HICO sits over the International Space Station (ISS) and is largely meant for scientific research related to the coastal studies, though it may be very well used for terrestrial applications. Table 1 briefly describes the HICO specifications.

Table 1: HICO specifications

\begin{tabular}{|c|c|}
\hline Parameter & Specification \\
\hline Spectral Range & $350-1080 \mathrm{~nm}$ \\
\hline $\begin{array}{c}\text { Spectral Channel } \\
\text { width (Normal } \\
\text { mode) }\end{array}$ & $5.7 \mathrm{~nm}$ \\
\hline SNR & $>200: 1$ for 5\% albedo target \\
\hline $\begin{array}{c}\text { Nadir crosstrack } \\
\text { GSD }\end{array}$ & $94 \mathrm{~m} @ 400 \mathrm{~km}$ \\
\hline $\begin{array}{c}\text { Nadir along-track } \\
\text { GSD }\end{array}$ & $99 \mathrm{~m}$ \\
\hline Scene size & $42 \mathrm{~km} \mathrm{x} 192 \mathrm{~km}$ \\
\hline Saturation & $\begin{array}{c}\text { Does not saturate when viewing } 95 \% \\
\text { albedo cloud }\end{array}$ \\
\hline
\end{tabular}

HICO data used in this study was dated $16^{\text {th }}$ December 2013. As shown in the FCC in figure 1, the image has many patches of clouds. Moreover, many non-water areas are also present. Both of these classes have to be removed before further analysis. So, three methods were investigated. First method was based on slicing the image based on the radiance, second was based on Normalized Difference Water Index (NDWI) and the third on Modified Normalized Difference Water Index (MNDWI). The most appropriate technique was used to remove the cloudy pixels and the non-water pixels.

\section{Masking of non-water area}

HICO data used for this study was available in DN, so was converted to radiance units using the specified gain settings. Since, large portion of the HICO scene had clouds apart from land mass, so cloud removal as well as removal of terrestrial parts was done using radiance slicing by applying a proper threshold. Also, NDWI and MNDWI were evaluated (Eq 1 and 1b). MNDWI (Xu, 2006) was particularly developed for removing the noise due to built-up land apart from background soil and vegetation effects.

$$
\begin{aligned}
& \text { NDWI }=(\text { Green-NIR }) /(\text { Green }+ \text { NIR }) \\
& \text { MNDWI }=(\text { Green-MIR }) /(\text { Green }+ \text { MIR })
\end{aligned}
$$

Once non-water areas from the image were masked, atmospheric correction was performed over the image using FLAASH module of ENVI image processing software. The reflectance image thus produced was used for bathymetry retrieval.

\section{Bathymetry retrieval}

Lyzenga (1978), in an extension to the Beer's law of light attenuation with increasing water depth, showed that the observed reflectance could be expressed by depth and bottom albedo as follows:

$$
R_{W}=\left(A_{d}-R_{\infty}\right) \exp (-g z)+R_{\infty}
$$

Where $R_{\infty}$ is the water column reflectance if the water were optically deep, $A_{d}$ is the bottom albedo, $z$ is the depth, and $g$ is a function of the diffuse attenuation coefficients for both downwelling and upwelling light (Stumpf et al, 2003). It implies that both the water depth as well as change in benthic type affects the reflectance spectrum in the range 480 to 610 $\mathrm{nm}$ (Loomis, 2009). Ma et al (2014) showed that if the influence of bottom albedo on the observed spectrum is removed, the remaining changes will all be caused by water depth variation. Pearson correlation coefficient (CC) and similarity coefficient (SC) can be used to derive bathymetry data from hyperspectral observations as follows (Ma et al 2014):

First, the reference spectrum coming from water depth closest to zero is selected. Then, the CC and SC at pixel level (i) are calculated as follows:

$$
C C \quad i=\frac{j_{j=1}^{N} R_{i, j}-\overline{R_{i}}\left(R_{0, j}-\overline{R_{0}}\right)}{\overline{j=1} R_{i, j}-{\overline{R_{i}}}^{2}} *{\overline{j=1} R_{0, j}-{\overline{R_{0}}}^{2}}_{N}^{N}+1
$$




$$
S C \quad i=\frac{{ }_{j=1}^{N}\left(R_{i, j} * R_{0, j}\right)}{\underset{j=1}{N} R_{i, j}^{2} *{\underset{j=1}{N} R_{0, j}}^{2}}+1
$$

In equations 3 and $4, N$ is total number of bands between 480 and $610 \mathrm{~nm} . R_{i, j}$ is the spectral reflectance of band $j$ at pixel $i$, $R_{0, j}$ is the reference spectral reflectance of band $j$. A constant in the formulae is added to ensure that the values are always positive.

From the above, water depth may be retrieved as:

$$
z i=k_{1} \frac{\ln (n S C i)}{\ln (n C C i)}-k_{0}
$$

Where $k 1$ is a tuneable constant to scale the ratio to depth, $n$ is a fixed constant that ensures the natural logarithms remain positive for every condition, and $k 0$ is the offset.

After the bathymetry estimates, the image was density sliced as per the variations in water depth. The spectra for each class was then retrieved and analyzed.

\section{RESULTS AND DISCUSSIONS}

HICO data was provided in the ISS format which is not compatible with the generally used image processing software like ENVI IDL. Hence, the data was converted to geotiff format using SeaDas software. The data was provided in the digital counts, so radiance image was generated using the provided scaling factors. The radiance image and corresponding spectral plots for major land-cover types is shown in Figure 2 below.

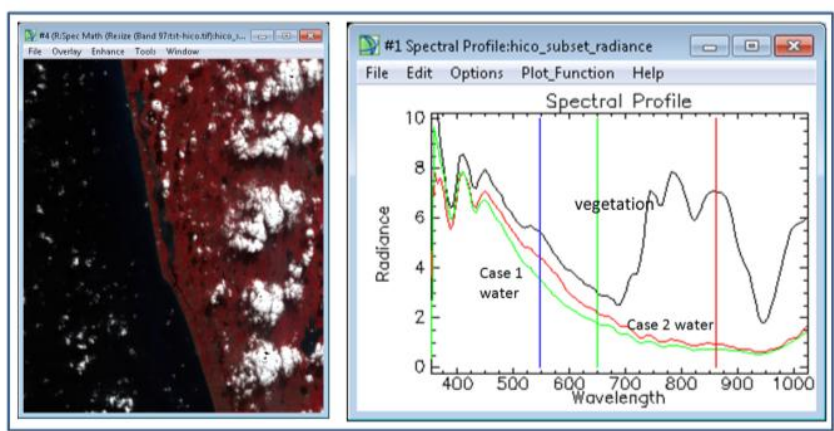

Figure 2: Radiance image of the study area with corresponding spectral plots. Note that unit of radiance is W/sq m/micrometer/steradian

Radiance plots for all the classes come in consensus with the observations from other sensors. A large amount of scattering in blue region is observed leading to an overall increase in radiance in the initial bands. Note that in both case 1 and case 2 waters not much spectral variability is observed in the radiance domain, although both the classes have immense difference in their constitution otherwise. The reflectance domain is expected to show the marked variability between the two classes. However, the prerequisite is masking of non-water regions before attempting reflectance retrieval. Hence, the radiance image was density sliced and NDWI and MNDWI maps were generated (figure 3).

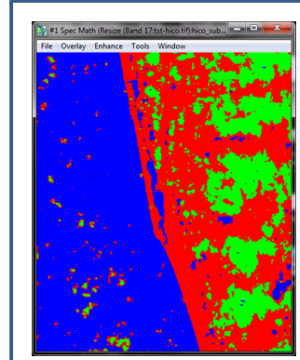

(a)

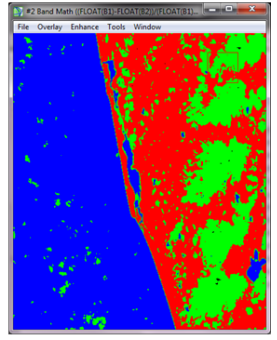

(b)

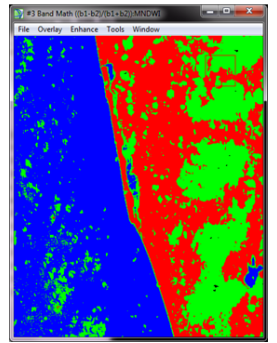

(c)

$$
\text { Water Land-mass Clouds }
$$

\section{Figure 3: Density sliced radiance, NDWI and MNDWI} images respectively

Broadly speaking, all the three methods of cloud making are able to mask out clouds an terrestrial areas. But, radiance based masking is highly subjective to the band slected for density slicing. So, this technique was dropped from comparison. When NDWI and MNDWI are carefully analyzed, it is observed that MNDWI outperforms the other two techniques of cloud masking as shown in figure 4.

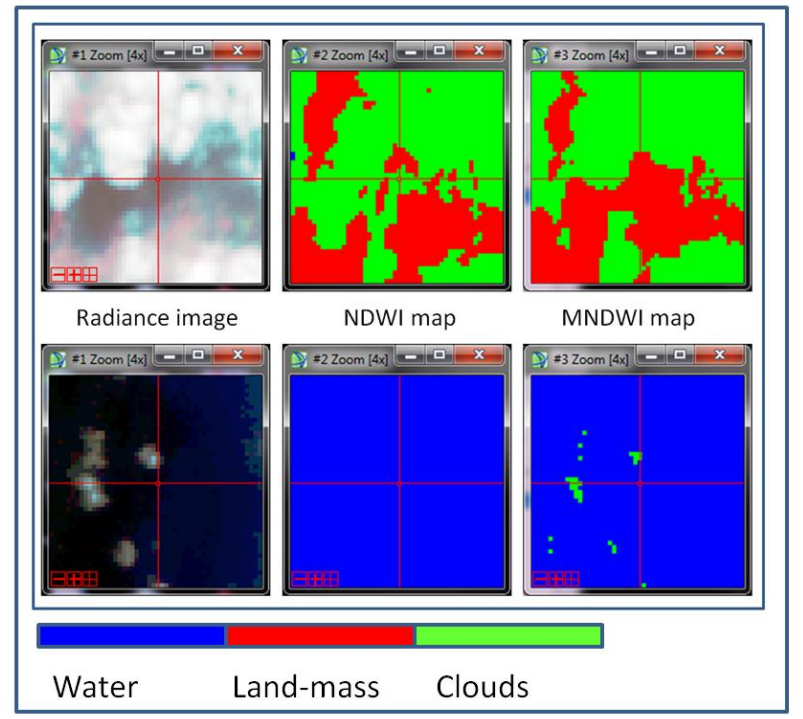

Figure 4: Cross Comparison of NDWI and MNDWI in segregating non-water regions

Note that in figure 4, FCC of first set of images show water body, which is shown as clouds in NDWI image while MNDWI suitably classifies it as non-clouds. Also, in second set of images, NDWI is not able to identify thin cloud patches but MNDWI classifies them suitably. Consequently, MNDWI based non-water masking was selected in this study. Xu (2006) aslo showed better performance of MNDWI over NDWI in cases where background noise is large.

As the sensor detected radiance is contaminated by solar backscatter from air molecules and aerosol, the procedure for atmospheric correction is necessary. Atmospheric correction 
was performed using FLAASH module of ENVI image processing software. Figure 5a shows the atmospherically corrected image of the study area while figure $5 \mathrm{~b}$ shows the MNDWI masked reflectance image of the study area.

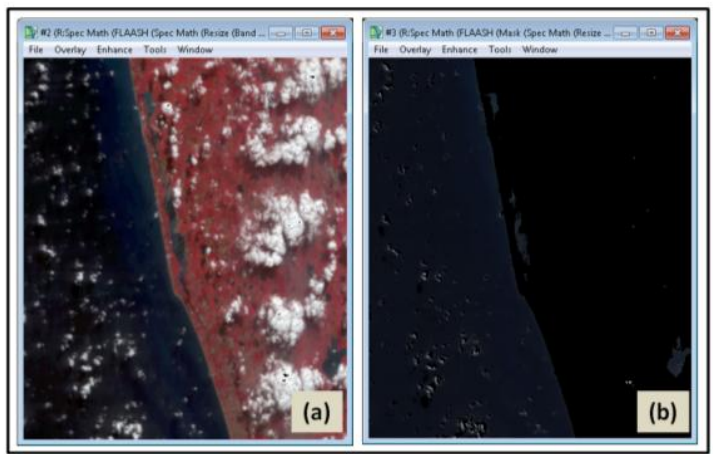

Figure 5: (a) Atmospherically corrected image of the study area (b) MNDWI based masked reflectance image displaying 'only water' regions

Corresponding to figure 5 , reflectance spectra were retrieved for case 1 as well as case 2 waters (shown in figure 6).

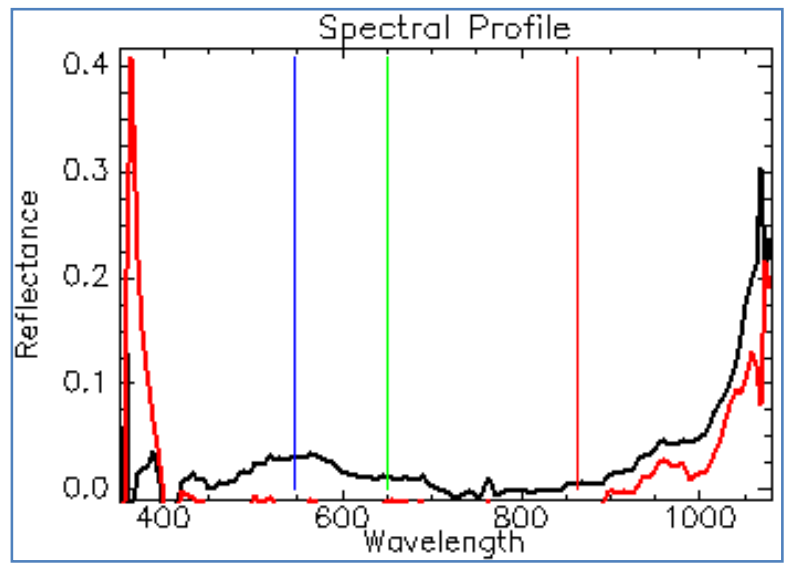

Figure 6: Reflectance plots for water

The reflectance goes to a high value of 0.4 or $40 \%$ in blue region, drops down to less than $5 \%$ and further low in higher wavelengths side.

In the two kinds of water, remarkable difference in magnitude as well as the pattern of the spectra is expected to be more pronounced in the wavelength region between 480 and 610nm. As a result, spectral plots within the same range are shown in figure 7 below. It may be noted that continuum removal was carried out to observe the distinct spectral variability between the two types of water.

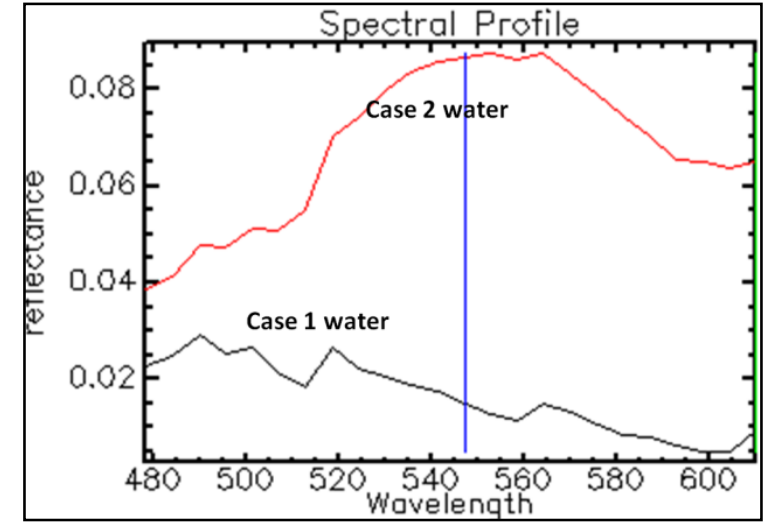

Figure 7: Continuum removed reflectance plots for case 1 and case 2 waters within the wavelength range of 480 and $610 \mathrm{~nm}$

As was expected, the reflectance for case 2 waters is higher than case 1 waters primarily due to the dissolved optically active substances in them. Note that case 2 waters show high reflectance in $\sim 550 \mathrm{~nm}$ region as is shown by green vegetation. This indicates the presence of phytoplanktons.

\section{Bathymetry Retrieval}

Bathymetry retrieval requires computations for the parameters $\mathrm{CC}$ and SC as per equations 3 and 4. Upon computing the two parameters and integrating them as in equation 5, bathymetry is retrieved (figure 8). Bathymetry map was density sliced into six classes showing water depth of $\sim-2 \mathrm{~m}$ (shown in yellow colour) to $<-10 \mathrm{~m}$ (shown in magenta colour).

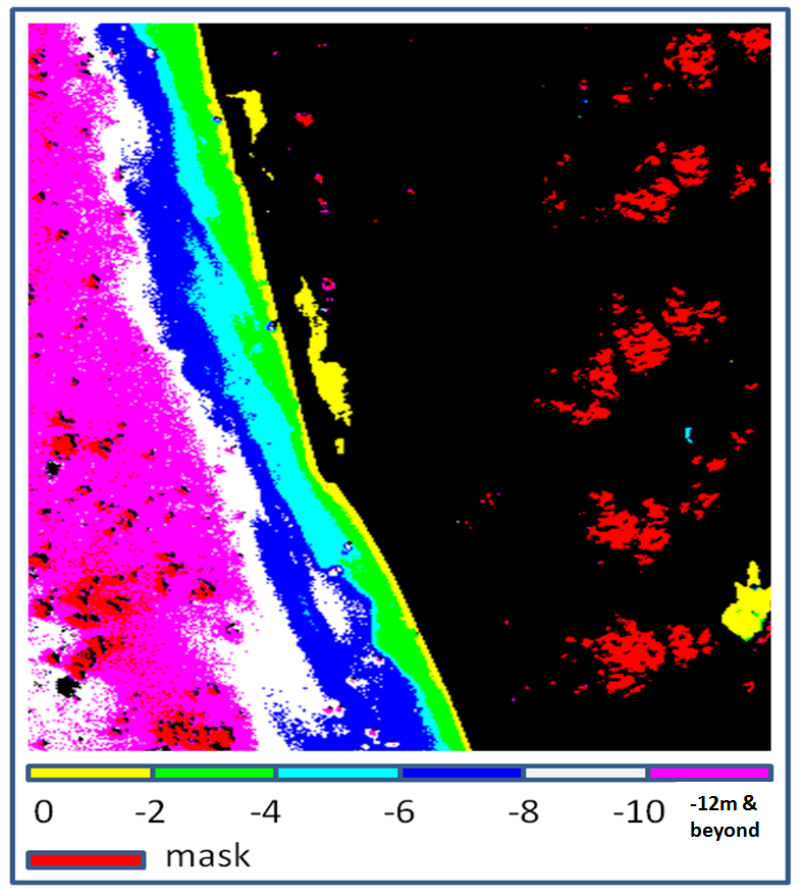

Figure 8: Density sliced bathymetry image

In order to observe the variability in depth on moving from inland water towards initiation of deep ocean, a horizontal 
transect was chosen and corresponding bathymetric plot was obtained (Figure 9). Inland waters and coastal areas appear to be shallow $(\sim 1-3 \mathrm{~m})$ than a sharp gradient in depth is observed followed by almost uniform depth.

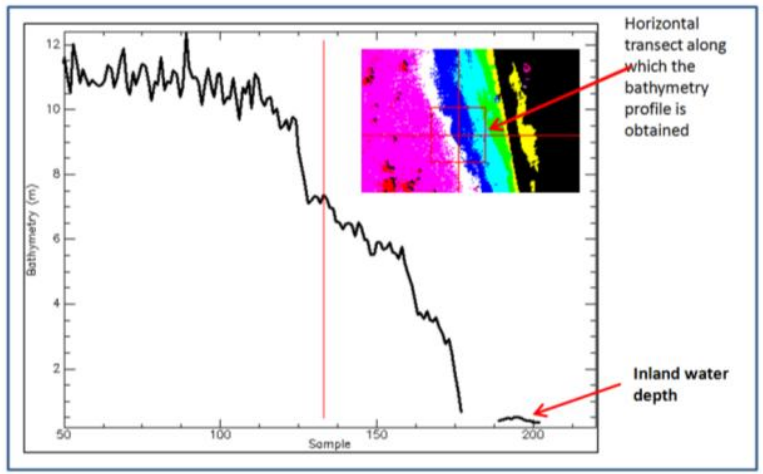

Figure 9: Bathymetry profile across the scene shown in inset

For each class of water the spectral plots were obtained and are shown in figure 10 below. It is evident that waters having variable depths show differences in their spectra also. Concurrent sample observations for chlorophyll content and suspended matter may be used to derive quantitative information from these spectra.

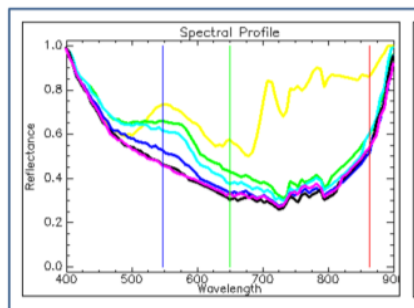

(a) Continuum removed spectra $400-900 \mathrm{~nm}$ range

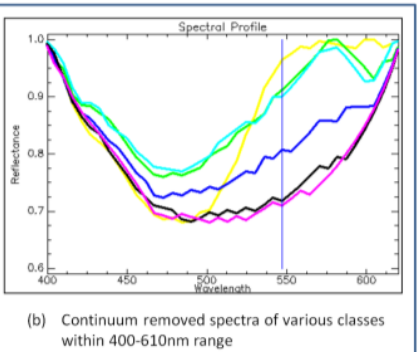

within 400-610nm range
Figure 10: Continuum removed reflectance spectra of the various classes of density sliced bathymetric image

\section{CONCLUSION}

In this study, hyperspectral data from HICO instrument is assessed for coastal studies. In this direction, the data was first processed for removing the terrestrial and clouds components by using three techniques. MNDWI was found to be the best when compared with the other two techniques. The radiance plots for different target types were extracted and were found to fall in consensus with the standard observations. The data was then used for retrieving shallow water bathymetry. Bathymetry estimates were found to vary from $\sim 1 \mathrm{~m}$ to $\sim 12 \mathrm{~m}$ in depth. The spectra of case 1 and case 2 waters obtained from the density sliced bathymetry image showed large variability in reflectance domain especially in the 480-610nm range. However, the reflectance values match well with the standard observations. This implies that both in radiance and reflectance domain, the spectral characteristics of water in term of pattern and amplitude show that HICO data can be very well used for quantitative coastal applications. The data can also be used for deriving shallow water bathymetry estimates useful for many navigation and climatic applications.

\section{References}

Conger, C. L. , E. J. Hochberg, C. H. Fletcher, and M. J. Atkinson. "Decorrelating remote sensing color bands from bathymetry in optically shallow waters," IEEE Trans. Geosci. Remote Sens., vol. 44, no. 6, pp. 1655-1660, Jun. 2006.

Cullen et al., 1997

http://www.ngdc.noaa.gov/mgg/global/ relief/ETOPO2/ETOPO2-2001

Loomis, M. J. "Depth derivation from the worldview-2 satellite using hyperspectral imagery," M.S. thesis, Dept. Meteorol., Naval Postgraduate School, Monterey, CA, USA, 2009.

Lyzenga, D. R. "Passive remotesensing techniques for mapping water depth and bottom features," Appl. Opt., vol. 17, no. 3, pp. 379-383, 1978.

Ma, Sheng, Zui Tao, Xiaofeng Yang, Yang Yu, Xuan Zhou, and Ziwei Li. "Bathymetry Retrieval from Hyperspectral Remote Sensing Data in Optical-Shallow Water", IEEE Transactions On Geoscience And Remote Sensing, Vol. 52, No. 2, February 2014.

Morel and Loisel, 1998

National Geophysical Data Center Data announcement 88$M G G-02$, Digital relief of the Surface of the Earth, Natl. Oceanic and Atmos. Admin., US Dept. Commerce, Boulder, Colorado, USA, 1988.

National Geophysical Data Center 2-minute Gridded Global Relief Data (ETOPO2v2), Natl. Oceanic and Atmos. Admin., U. S. Dept. of Commerce, (http://www.ngdc.noaa.gov/mgg/fliers/06mgg01.html), 2006.

Nayak Shailesh, P Chauhan, H.B. Chauhan, Anjali Bahuguna and A. N arendra Nath.'IRS-1C applications for Coastal Zone Mangement”, Current Science, Vol 70, No. 7, April 1996.

Stumpf, R. P., K. Holderied, and M. Sinclair. "Determination of water depth with high-resolution satellite imagery over variable bottom types," Limnol. Oceanogr., vol. 48, no. 1, pp. 547-556, Jan. 2003.

$\mathrm{Xu}$, Hanqiu. "Modification of normalised difference water index (NDWI) to enhance open water features in remotely sensed imagery", Int J of Remote Sensing, Vol. 27, No. 14, 3025-3033, July 2006. 